\title{
Supporting Information Dynamical Manifestations of Supercooling in Amyloid Hydration
}

\author{
Priti Roy ${ }^{\#}$, Sneha Menon ${ }^{\$}$ and Neelanjana Sengupta ${ }^{\#}$ \\ \#Department of Biological Sciences, Indian Institute of Science Education and Research Kolkata, \\ Mohanpur, West Bengal, India, 741246
}

${ }^{\$}$ Tata Institute of Fundamental Research Hyderabad, Telangana, India, 500046 


\section{Figures}

1. Thermal trend of $\mathrm{S}_{\mathrm{SC}} \quad \mathrm{S} 3$

2. MSD time evolution of PHS and protein $\quad$ S4

3. Diffusive regime $\langle\mathrm{MSD}\rangle \quad$ S5

4. Van Hove function, $G_{S}(r ; t)$ of BW S6

5. Van Hove function, $G_{S}(r ; t)$ of PHS $\quad S 7$

6. Non-Gaussian parameter (NGP) of BW S8

7. Non-Gaussian parameter (NGP) of PHS S9

8. Rotational relaxation of BW $\quad \mathrm{S} 10$

9. Thermal trend of PHS rotational relaxation parameters $\quad$ S11

10. Power spectra of BW $\quad$ S12

11. Vibrational modes of BW $\quad$ S13

$\begin{array}{lr}\text { 12. Vibrational modes of PHS } & \text { S14 }\end{array}$

\section{Table}

1. Protein side-chain entropy $\quad$ S15

2. Mean squared displacement for protein and PHS S16

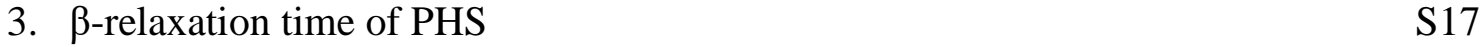

4. Mean relaxation time $(<\tau>)$ and heterogeneity $(\beta)$ for the BW and PHS S18

$\begin{array}{lr}\text { 5. } \omega_{1}, \omega_{2} \text { and } \omega_{3} \text { of BW and PHS } & \text { S19 }\end{array}$

$\begin{array}{ll}\text { 6. ANOVA analysis of spectral bands } & \text { S20 }\end{array}$ 


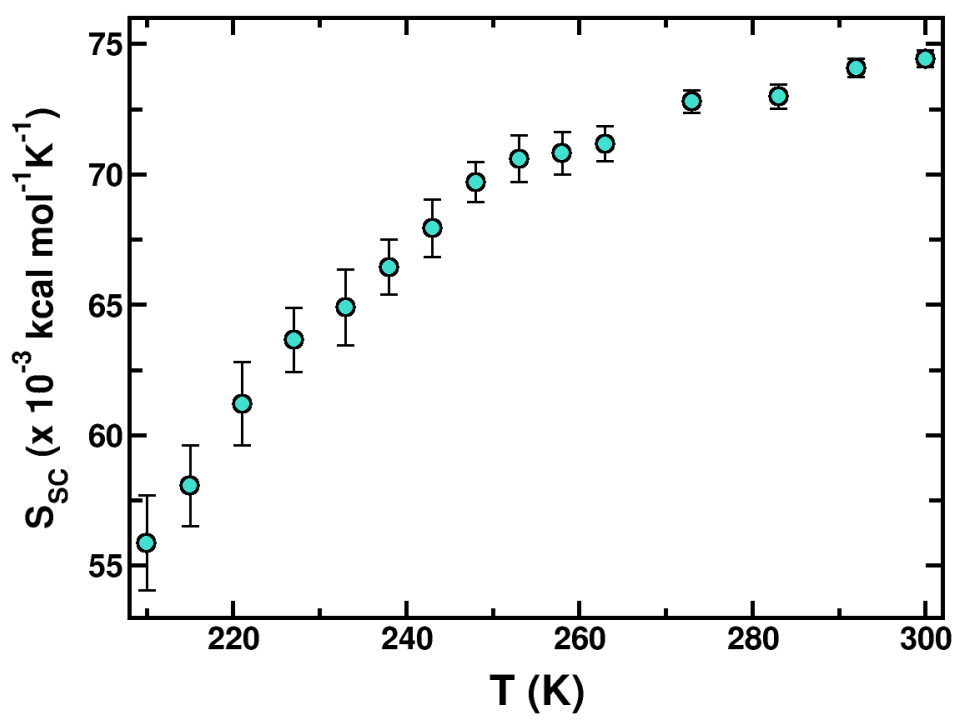

Figure S1. Thermal trend of side chain conformational entropy $\left(\mathrm{S}_{\mathrm{SC}}\right)$. The error bars are calculated from the analyses over the last $800 \mathrm{~ns}$ of simulation for each temperature. All the data tabulated in Table S1. See main-text for details. 

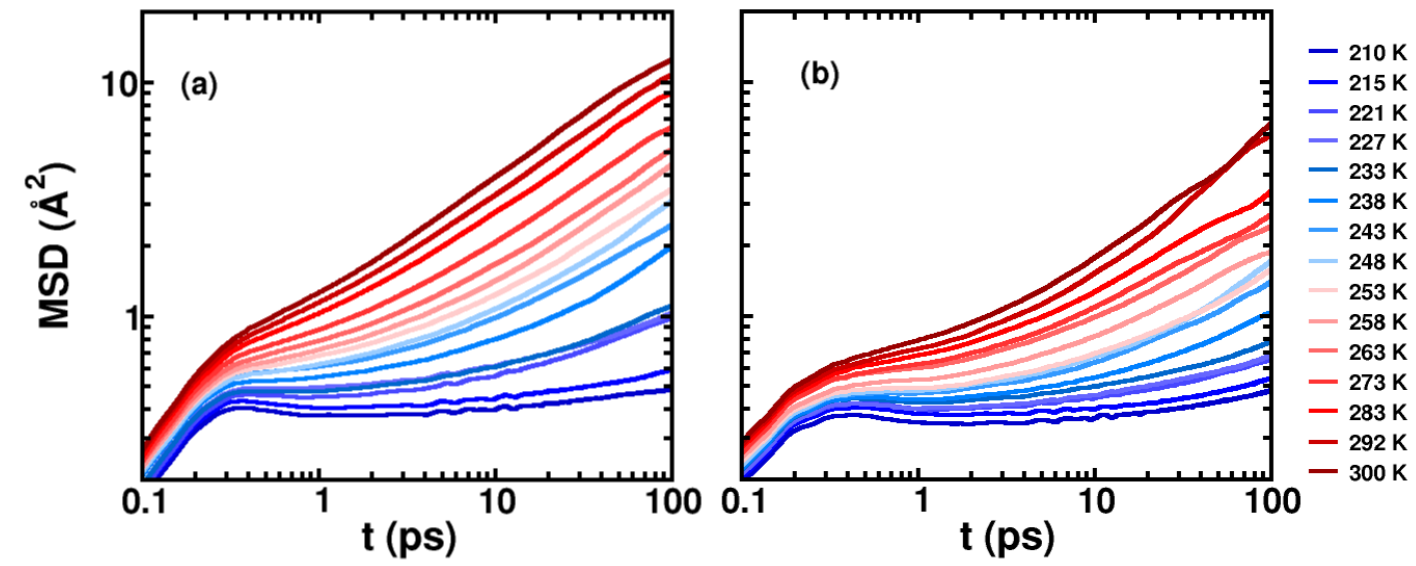

Figure S2. Mean squared displacement of PHS (a) and protein (b). The time evolution of MSD is averaged over three replicas of simulation. 

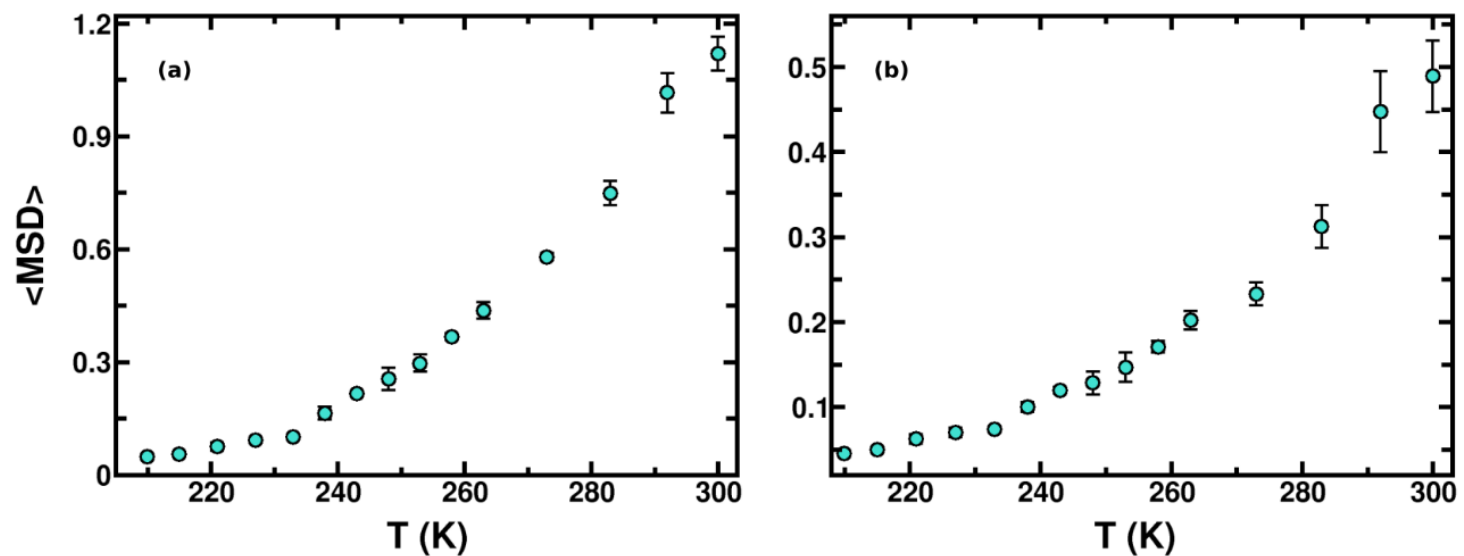

Figure S3. Thermal trend of diffusive regime (average) mean-squared displacement $(\langle\mathrm{MSD}\rangle$ ) of hydration water (a) and amyloid (b). The $\langle$ MSD $\rangle$ and error bars are calculated from the analyses on three independent trajectories. All the data tabulated in Table S2. See main-text for details. 

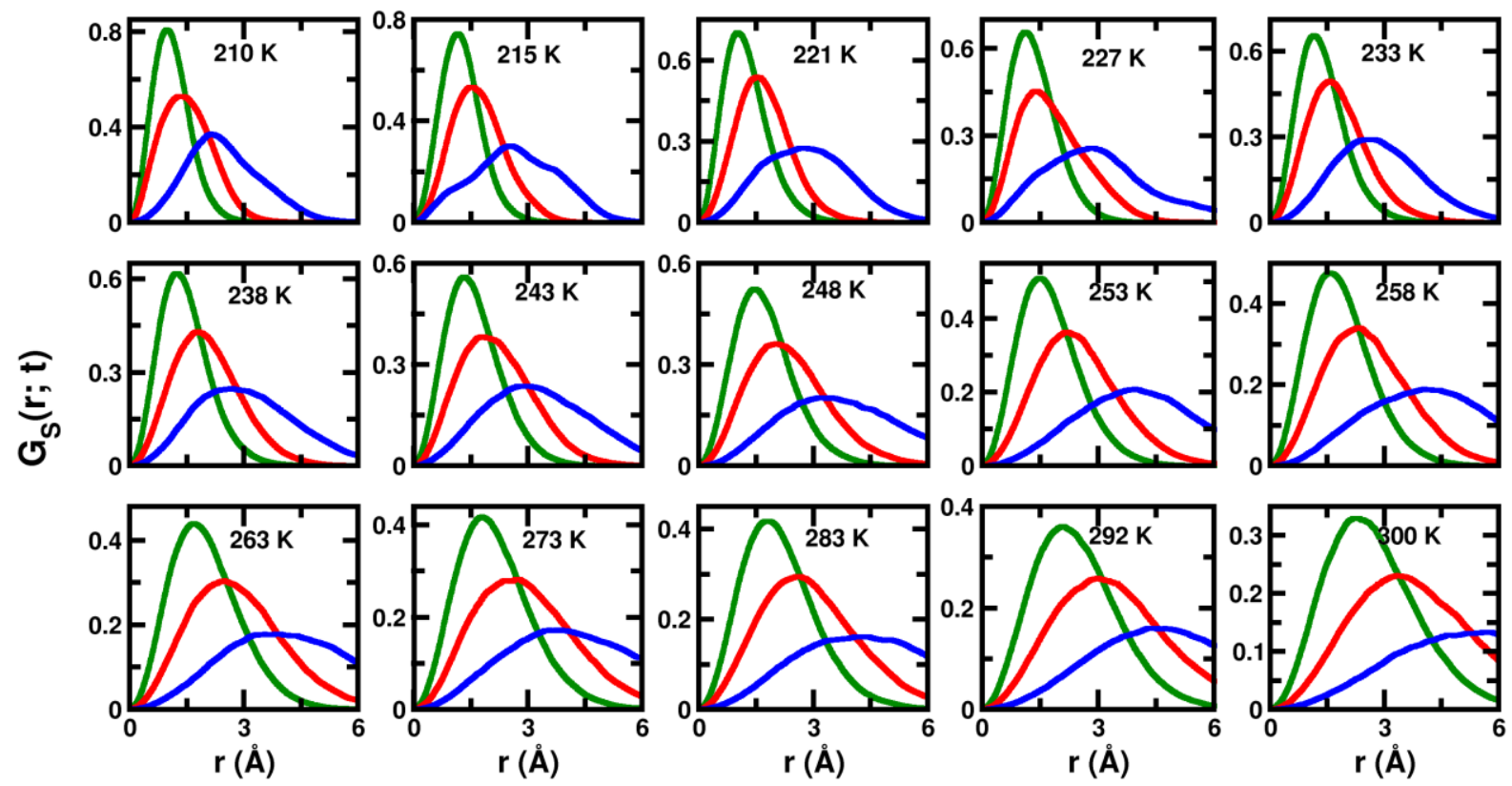

Figure S4. The van Hove self-correlation function for bulk water of the correlation time 5ps (green), 10ps (red) and 25ps (blue). 


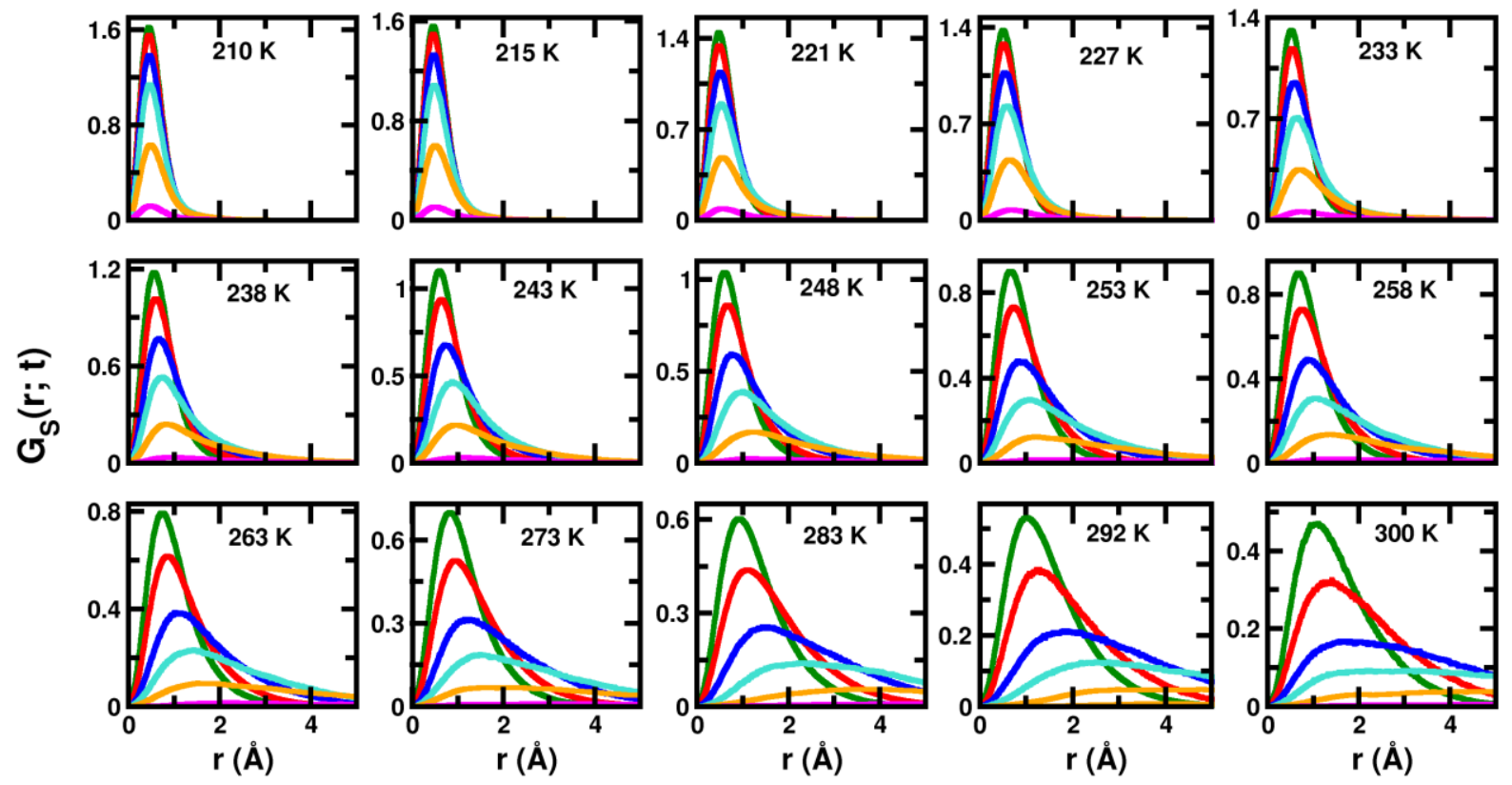

Figure S5. The van Hove self-correlation function for PHS of the correlation time 5ps (green), 10ps (red) 25ps (blue), 50 ps (cyan), 100 ps (orange) and 150 ps (magenta). 


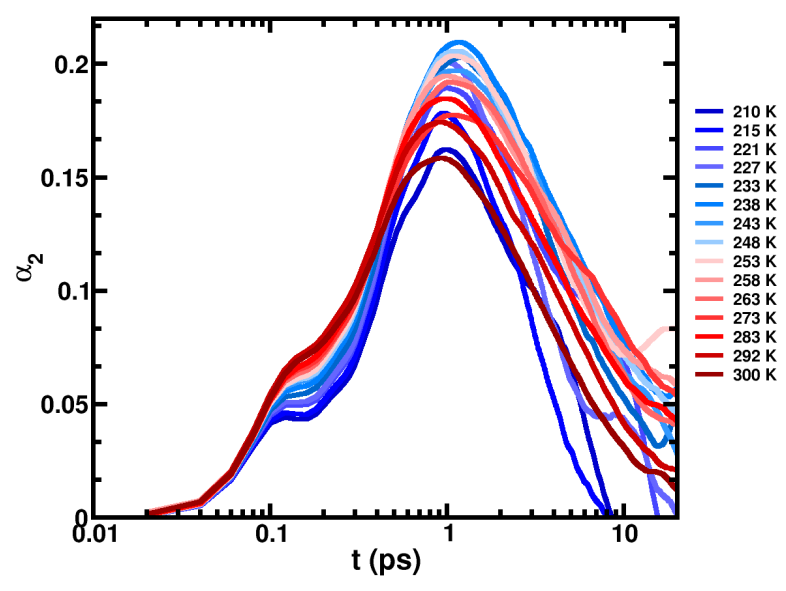

Figure S6. Time evolution of the non-Gaussian parameter (NGP) for bulk water (BW). 

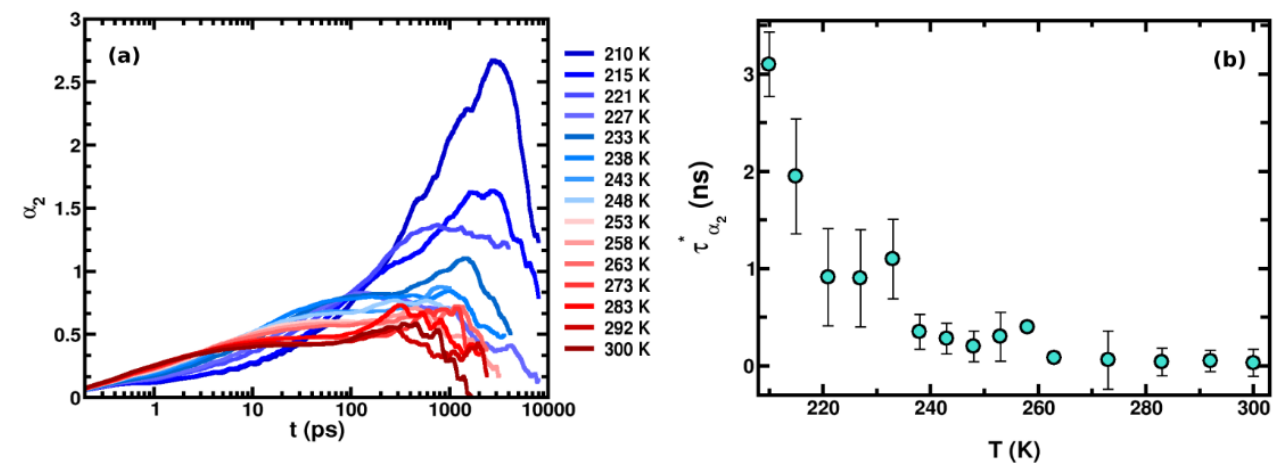

Figure S7. Time evolution of the non-gaussian parameter for PHS water (a) and thermal trend of $\beta$-relaxation time $\left(\tau_{\alpha 2}{ }^{*}\right)$. The presented data averaged over three independent simulations of amyloid assembly and the error bars are calculated from the analyses of three simulations. See main-text for details. The data of $\tau_{\alpha 2}{ }^{*}$ tabulated in Table S3. 

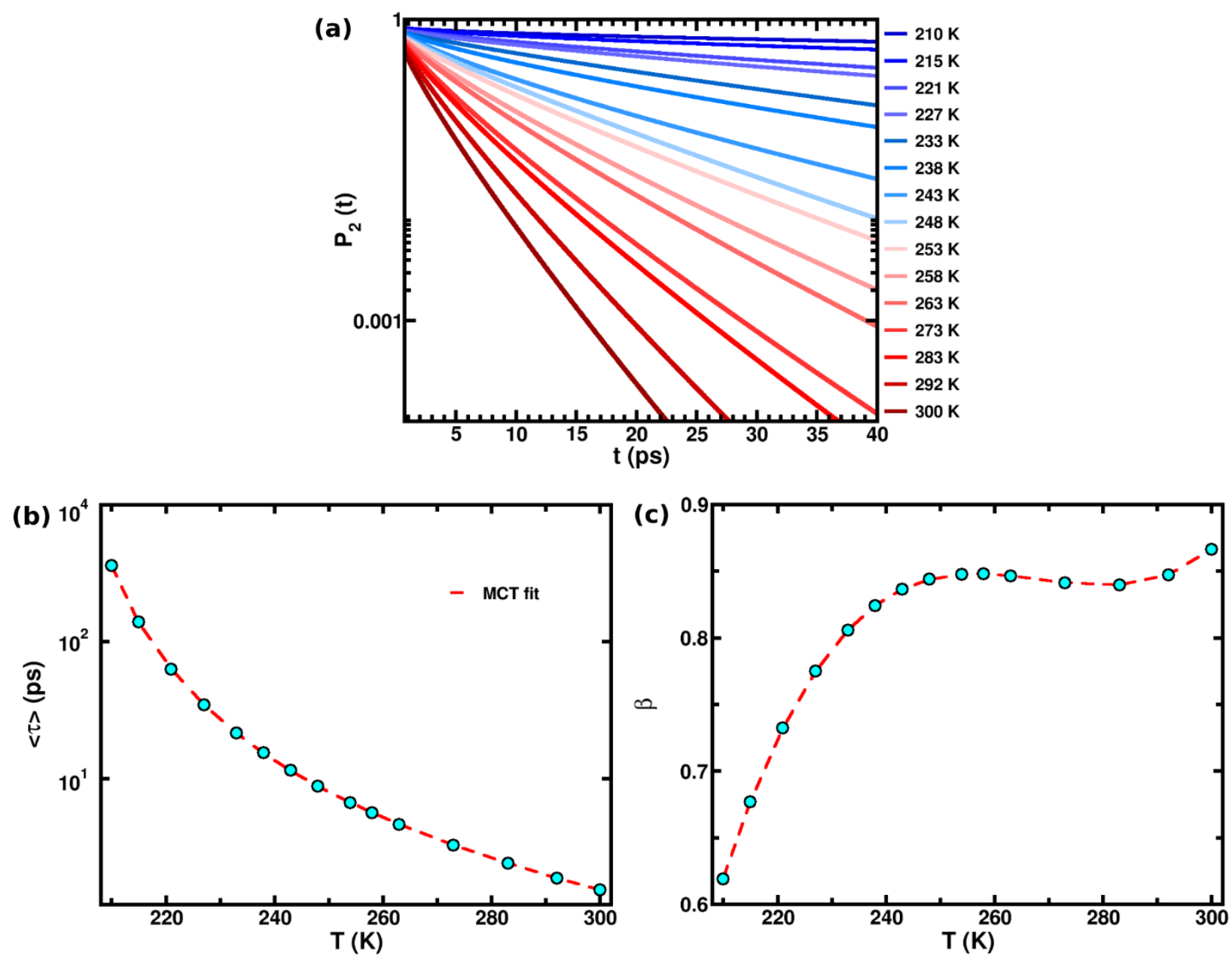

Figure S8. Time-correlation function of the rotational relaxation $\left(P_{2}(t)\right)$ of BW for each temperature (a). Presented $P_{2}(t)$ is averaged over the three independent trajectories (see method for detail). The fitted data of mean relaxation time $(<\tau>)$ and rotational heterogeneity $(\beta)$ is shown in (b) and (c) respectively. 

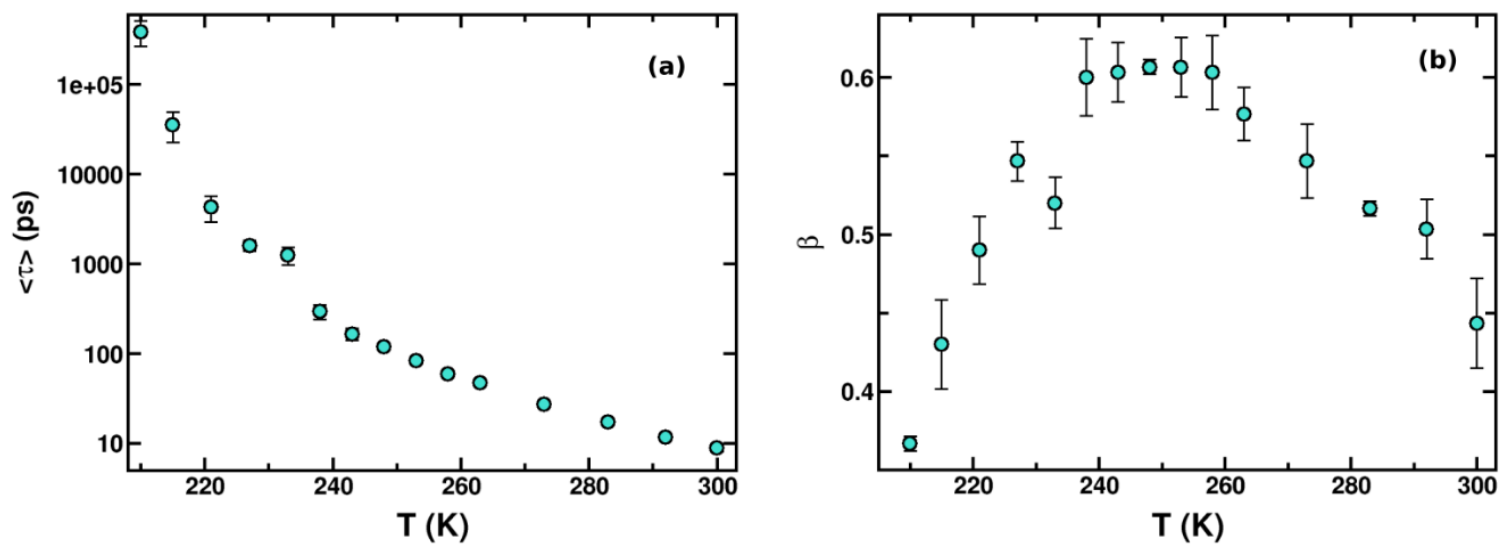

Figure S9. Thermal signature of rotational relaxation time $(<\tau>$; (a)) and heterogeneity parameter $(\beta$; (b)) of PHS. The presented averages and error bars are calculated over three independent simulations of amyloid assembly. See main-text for details. All the data tabulated in Table S4. 

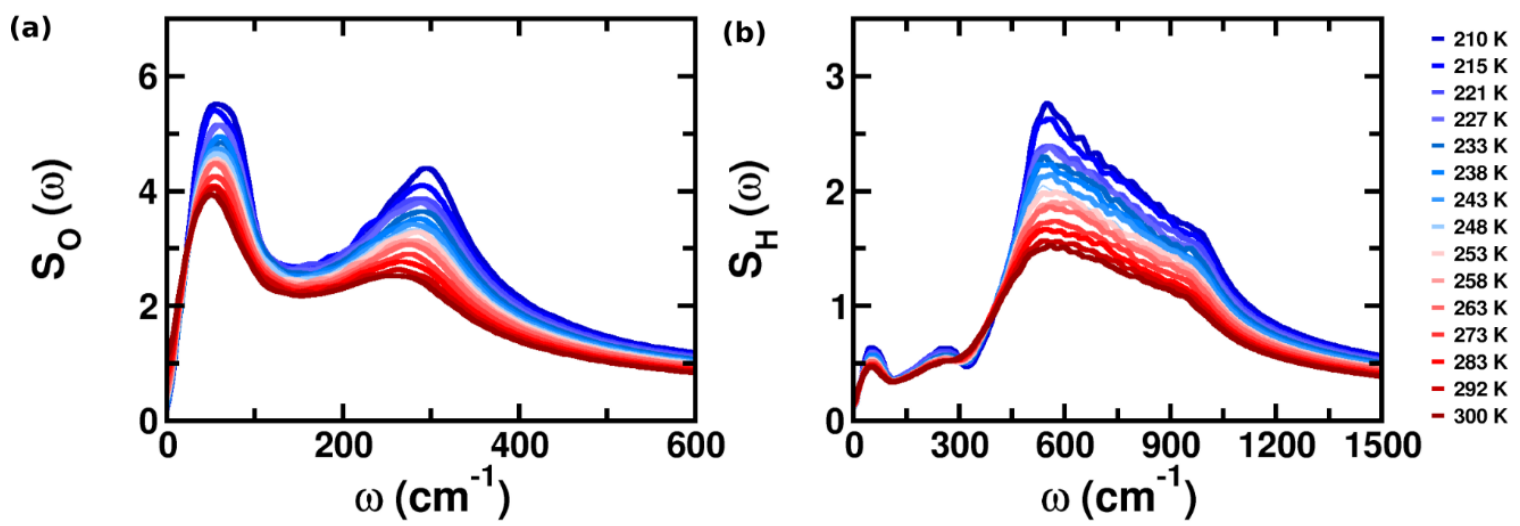

Figure $S 10$. The power spectra of bulk water oxygen $\left(S_{O}(\omega)\right.$; left panel) and hydrogen $\left(S_{H}(\omega)\right.$; right panel) atom generated by Fourier Transformation of VACF of respected atom. See main-text for details. 

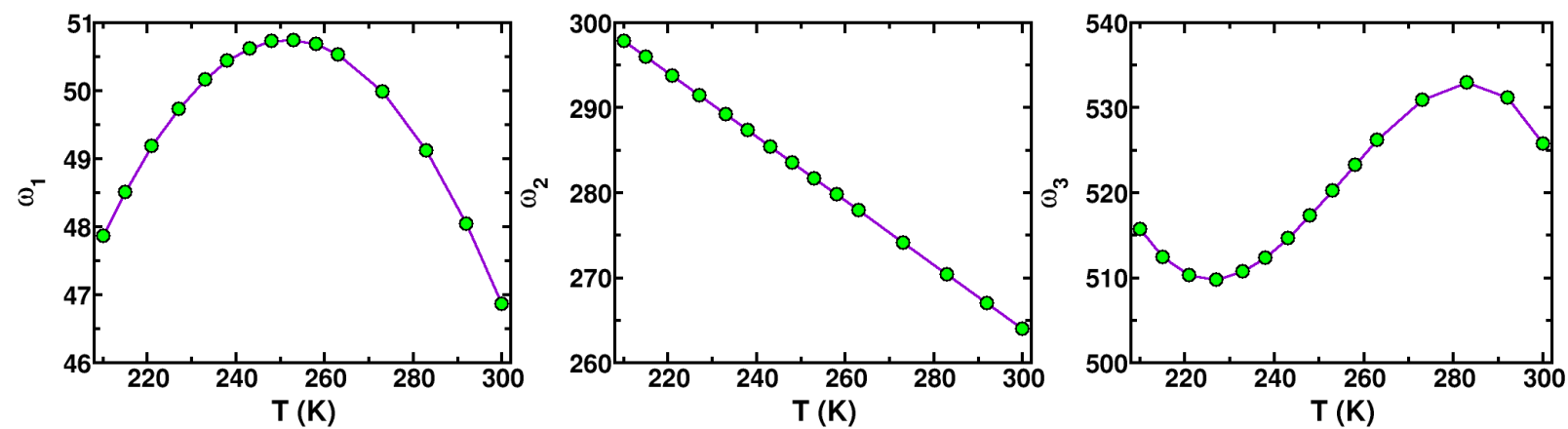

Figure S11. Representation of the vibrational modes extracted from the power spectra of bulk water: $\omega_{1}$ (left panel), $\omega_{2}$ (middle panel) and $\omega_{3}$ (right panel). For detail see the method and main text. Here depicted data points are regression fit of measured average value of three trajectories (see Table S5). 

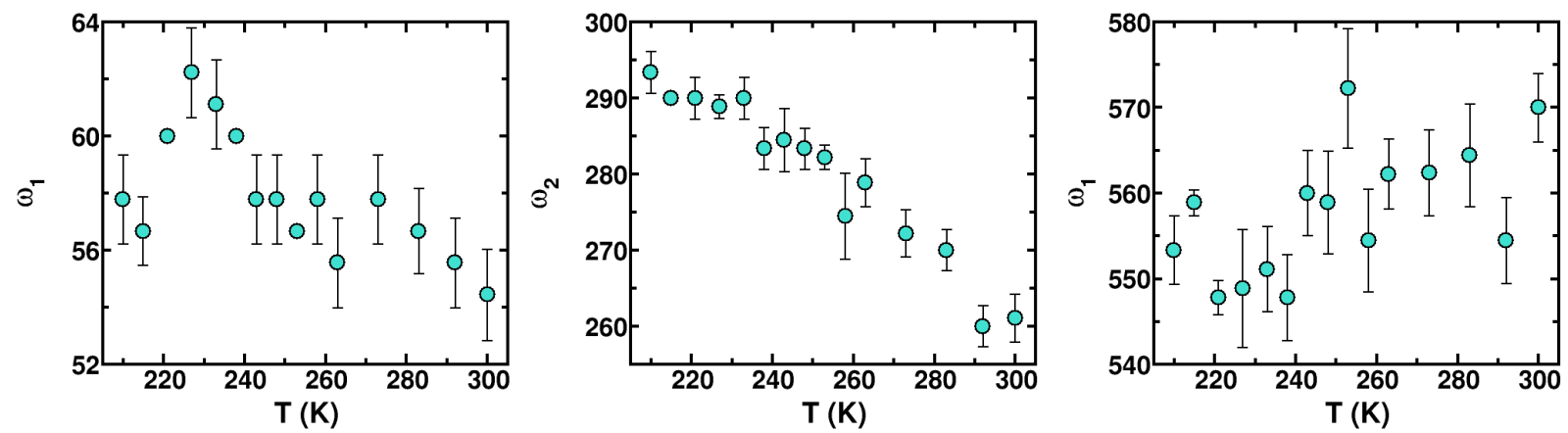

Figure S12. Thermal signature of vibrational modes of PHS. The presented averages and error bars are calculated over three independent simulations of amyloid assembly. All the data tabulated in Table S5. See main-text for details. 


\begin{tabular}{|c|c|}
\hline $\begin{array}{c}\text { Temperature } \\
(\mathrm{K})\end{array}$ & Sidechain entropy $\left(\mathrm{S}_{\mathrm{SC}}\right)$ \\
\hline 210 & $55.88(1.81)$ \\
\hline 215 & $58.06(1.55)$ \\
\hline 221 & $61.21(1.61)$ \\
\hline 227 & $63.65(1.24)$ \\
\hline 233 & $64.91(1.45)$ \\
\hline 238 & $66.45(1.05)$ \\
\hline 243 & $67.93(1.1)$ \\
\hline 248 & $69.71(0.76)$ \\
\hline 253 & $70.59(0.89)$ \\
\hline 258 & $70.81(0.80)$ \\
\hline 263 & $71.18(0.67)$ \\
\hline 273 & $72.79(0.44)$ \\
\hline 283 & $72.98(0.47)$ \\
\hline 292 & $74.08(0.35)$ \\
\hline 300 & $74.43(0.33)$ \\
\hline
\end{tabular}

Table S1. The calculated protein side-chain entropy for the temperature range 210-300 K. For the methods, see the main text. 


\begin{tabular}{|c|c|c|}
\hline Temperature & Mean squared & slacement $\left(\AA^{2}\right)$ \\
\hline$(\mathrm{K})$ & $\mathrm{PHS}^{\mathrm{O}}$ & Protein $^{\mathrm{H}}$ \\
\hline 210 & $0.049(0.001)$ & $0.046(0.002)$ \\
\hline 215 & $0.056(0.001)$ & $0.05(0.001)$ \\
\hline 221 & $0.075(0.011)$ & $0.062(0.006)$ \\
\hline 227 & $0.092(0.004)$ & $0.07(0.002)$ \\
\hline 233 & $0.101(0.002)$ & $0.074(0.002)$ \\
\hline 238 & $0.164(0.017)$ & $0.1(0.005)$ \\
\hline 243 & $0.217(0.003)$ & $0.12(0.004)$ \\
\hline 248 & $0.256(0.03)$ & $0.128(0.014)$ \\
\hline 253 & $0.297(0.023)$ & $0.147(0.017)$ \\
\hline 258 & $0.368(0.009)$ & $0.171(0.007)$ \\
\hline 263 & $0.437(0.022)$ & $0.202(0.011)$ \\
\hline 273 & $0.58(0.009)$ & $0.233(0.013)$ \\
\hline 283 & $0.749(0.032)$ & $0.312(0.025)$ \\
\hline 292 & $1.016(0.052)$ & $0.447(0.047)$ \\
\hline 300 & 1.119 (0.044) & $0.489(0.042)$ \\
\hline
\end{tabular}

Table S2. The measured value of mean squared displacement for protein and PHS of considered temperature. Standard deviation provided within the parenthesis. For protein and PHS, the analysis is done by considering hydrogen $(\mathrm{H})$ and oxygen $(\mathrm{O})$ atom respectively marked with superscript. See main text for details. 


\begin{tabular}{|c|c|}
\hline Temperature (K) & $\tau^{*}{ }_{a 2}(\mathrm{~ns})$ \\
\hline 210 & $3.1(0.33)$ \\
\hline 215 & $1.95(0.59)$ \\
\hline 221 & $0.91(0.5)$ \\
\hline 227 & $0.9(0.5)$ \\
\hline 233 & $1.1(0.41)$ \\
\hline 238 & $0.35(0.18)$ \\
\hline 243 & $0.28(0.24)$ \\
\hline 248 & $0.2(0.16)$ \\
\hline 253 & $0.3(0.25)$ \\
\hline 258 & $0.4(0.02)$ \\
\hline 263 & $0.08(0.03)$ \\
\hline 273 & $0.06(0.3)$ \\
\hline 283 & $0.04(0.14)$ \\
\hline 292 & $0.05(0.11)$ \\
\hline 300 & $0.03(0.14)$ \\
\hline
\end{tabular}

Table S3. The $\beta$-relaxation time of PHS water obtained from the time evolution of NGP (see text for details); $\tau^{*}{ }_{2}$, time corresponding to the peak of NGP (see Fig. S4). Standard deviation mentioned within the parenthesis. 


\begin{tabular}{|c|c|c|c|c|}
\hline \multirow{2}{*}{$\begin{array}{c}\text { Temperature } \\
\text { K) }\end{array}$} & \multicolumn{2}{|c|}{$<\tau>(\mathrm{ps})$} & \multicolumn{2}{|c|}{$\beta$} \\
\cline { 2 - 5 } & BW & PHS & BW & PHS \\
\hline 210 & 360.54 & $387472.32(122391.98)$ & 0.6 & $0.37(0.01)$ \\
\hline 215 & 142.05 & $35741.36(12345.66)$ & 0.67 & $0.43(0.03)$ \\
\hline 221 & 53.23 & $4314.4(1405.89)$ & 0.77 & $0.49(0.02)$ \\
\hline 227 & 42.11 & $1600.46(216.49)$ & 0.78 & $0.55(0.02)$ \\
\hline 233 & 22.92 & $1244.92(278.24)$ & 0.86 & $0.6(0.02)$ \\
\hline 238 & 16.3 & $294.64(53.51)$ & 0.77 & $0.6(0.02)$ \\
\hline 243 & 9.53 & $166.15(25.8)$ & 0.78 & $0.61(0.02)$ \\
\hline 248 & 8.12 & $118.27(2.93)$ & 0.87 & $0.61(0.01)$ \\
\hline 253 & 6.91 & $83.57(6.38)$ & 0.66 & $0.61(0.02)$ \\
\hline 258 & 5.22 & $59.3(3.37)$ & 0.84 & $0.6(0.02)$ \\
\hline 263 & 4.54 & $47.09(2.21)$ & 0.85 & $0.58(0.02)$ \\
\hline 273 & 3.31 & $27.2(1.8)$ & 0.84 & $0.55(0.02)$ \\
\hline 283 & 3.01 & $17.26(0.42)$ & 0.87 & $0.52(0.01)$ \\
\hline 292 & 2.38 & $11.72(0.66)$ & 0.85 & $0.5(0.02)$ \\
\hline 300 & 1.86 & $8.93(0.81)$ & & $0.44(0.03)$ \\
\hline & & & & \\
\hline & & & & \\
\hline
\end{tabular}

Table S4. The mean relaxation time $(<\tau>)$ and heterogeneity $(\beta)$ for the BW and PHS; extracted by using KWW fit over the rotational relaxation (see main text). In the PHS, the standard deviation is provided in braces which obtained from three independent trajectories. 


\begin{tabular}{|c|c|c|c|c|c|c|}
\hline \multirow{2}{*}{$\begin{array}{c}\text { Temperature } \\
\text { (K) }\end{array}$} & \multicolumn{3}{|c|}{ BW } & \multicolumn{3}{|c|}{ PHS } \\
\hline & $\omega_{1}$ & $\omega_{2}$ & $\omega_{3}$ & $\omega_{1}$ & $\omega_{2}$ & $\omega_{3}$ \\
\hline 210 & 49.99 & 303.3 & 523.39 & $57.78(1.57)$ & $293.32(2.75)$ & $553.32(7.18)$ \\
\hline 215 & 49.66 & 289.97 & 503.38 & $56.66(2.72)$ & 289.97 & $558.87(1.52)$ \\
\hline 221 & 49.99 & 289.97 & 503.38 & $59.99(0.0)$ & $289.97(2.72)$ & 547.76 (5.7) \\
\hline 227 & 49.99 & 293.3 & 506.71 & $62.22(1.57)$ & $288.86(1.57)$ & 548.87 (6.89) \\
\hline 233 & 46.66 & 286.64 & 523.39 & $61.11(1.57)$ & $289.97(2.72)$ & $551.09(8.27)$ \\
\hline 238 & 49.99 & 279.97 & 520.05 & $59.99(0.0)$ & $283.32(2.74)$ & $547.76(13.65)$ \\
\hline 243 & 49.99 & 293.3 & 530.05 & $57.77(1.57)$ & $284.42(4.16)$ & $559.87(10.89)$ \\
\hline 248 & 49.99 & 289.97 & 493.38 & $57.77(1.57)$ & $283.31(2.72)$ & $558.87(13.38)$ \\
\hline 253 & 53.34 & 279.97 & 510.05 & $56.6(0.0)$ & $282.19(1.57)$ & $572.2(19.32)$ \\
\hline 258 & 53.34 & 279.92 & 533.39 & $57.77(1.57)$ & $274.42(5.66)$ & $554.43(12.32)$ \\
\hline 263 & 53.34 & 279.97 & 520.05 & $55.55(1.57)$ & $278.86(3.14)$ & $562.2(4.11)$ \\
\hline 273 & 46.66 & 279.97 & 533.39 & $57.77(1.57)$ & $272.2(3.14)$ & $562.35(8.21)$ \\
\hline 283 & 46.66 & 263.31 & 546.72 & $56.66(2.72)$ & $269.97(2.72)$ & $564.42(14.95)$ \\
\hline 292 & 49.99 & 266.64 & 516.72 & $55.55(1.57)$ & $259.97(2.72)$ & $554.42(10.25)$ \\
\hline 300 & 46.66 & 263.31 & 530.05 & $54.44(3.14)$ & $261.09(3.14)$ & $569.98(7.15)$ \\
\hline
\end{tabular}

Table S5. Tabulation of low frequency band position of BW and PHS. In the PHS, the standard deviation is provided in braces which obtained from three independent trajectories. See main text for details. 


\begin{tabular}{|c|c|c|c|c|c|}
\hline \multirow{2}{*}{ Band } & $\begin{array}{c}\text { F } \\
\text { Value }\end{array}$ & \multicolumn{2}{|c|}{ Degrees of Freedom (DF) } & \multicolumn{2}{|c|}{$F_{\mathrm{c}}$ value } \\
\hline$\omega_{1}$ & 2.98 & Among Groups & Within Groups & at $\alpha=0.05$ & at $\alpha=0.1$ \\
\cline { 1 - 2 }$\omega_{2}$ & 4.47 & \multirow{2}{*}{14} & 30 & 2.31 & 1.91 \\
\cline { 1 - 2 }$\omega_{3}$ & 2.97 & & & & \\
\hline
\end{tabular}

Table S6. ANOVA analysis of vibrational mode $(\omega)$ to determine the statistical significance of temperature effect based on the $\mathrm{F}$ test. We have studied 15 temperatures (Groups; $\mathrm{k}$ ) from $210 \mathrm{~K}$ to $300 \mathrm{~K}$, and in each group, we have three data points $(\mathrm{n}=15 * 3=45)$. The degrees of freedom of the groups are calculated as (k-1), whereas within the groups is done by $(n-k)$. Our null hypothesis $\left(\mathrm{H}_{0}\right)$ is that temperature does not influence $\omega$, and the means of each population (temperature) will be equal. For each type of vibrational mode, we found that the $\mathrm{F}$ value is greater than the critical $\mathrm{F}(\mathrm{Fc})$ value, and thus, we reject $\mathrm{H} 0$. The data provide sufficient evidence, at the level of $5 \%$ or $10 \%$ of significance, to conclude that the means of all studied temperatures are not equal. 